\title{
Gold Coated Glass in the Building Industry
}

\section{THE DESIGN OF INTERFERENCE SYSTEMS FOR HEAT REFLECTION}

\section{Rolf Groth}

Flachglas AG Delog•Detag, Fürth, West Germany

\section{and Walter Reichelt}

WC Heraeus GmbH, Hanau, West Germany

Thin gold films on window panes reflect infra-red radiation and transmit a proportion of visible light that is dependent on the thickness of coating, so providing an effective means of protection from solar radiation. Gold films on double-glazed windows have the further advantage of reducing heat losses in winter, so reducing fuel costs.

In modern office buildings large areas of glass are used to give an even distribution of light and, in the case of large open-plan offices, to give workers an uninterrupted view and a sense of contact with the outside world. Such large window areas have, however, created problems. Intense sunlight causes overheating in these offices, necessitating extensive air-conditioning.

Ordinary window glass is almost completely transparent to radiation extending from the ultraviolet to the infra-red. This range represents the spectrum of solar radiation received at the earth's surface.

Figure 1 shows the distribution of solar energy given by Moon's air mass, where $\mathrm{m}=2$ (1), corresponding to an angle of incidence of $30^{\circ}$ to the horizontal. Of the total radiant energy about 3 per cent is in the ultra-violet range. $(\lambda<0.4 \mu \mathrm{m}), 51$ per cent in the visible range $(\lambda=0.4$ to $0.75 \mu \mathrm{m})$, and 46 per cent in the infra-red range. The distribution of solar spectral energy is dependent partiy on the height of the sun, and partly on the moisture con-

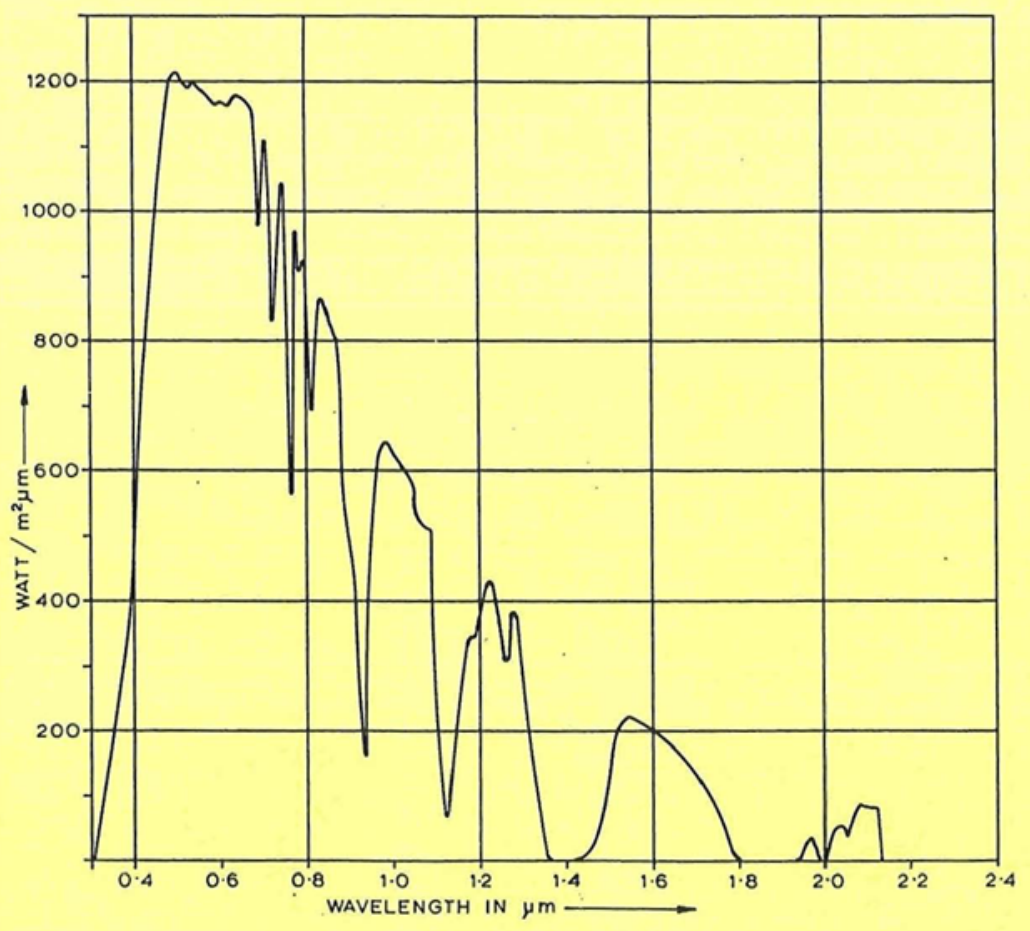

Fig. I The spectral distribution of solar energy. Of the total radiant energy in this example about 3 per cent is in the ultra-violet range, below $0.4 \mu \mathrm{m}, 51$ per cent in the visible range, frem 0.4 to $0.75 \mu \mathrm{m}$, while $\mathbf{4 6}$ per cent is in the infra-red 


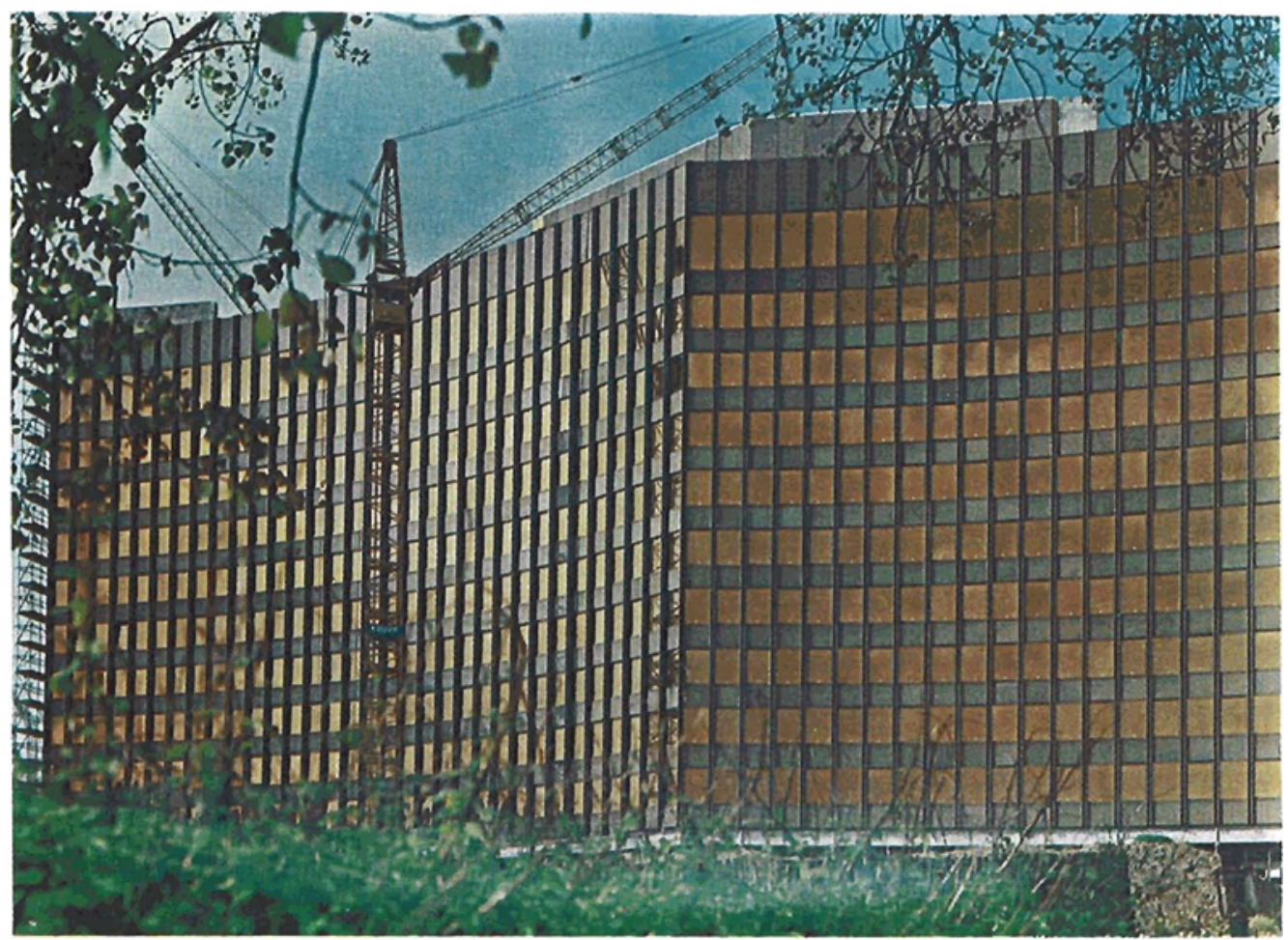

A sultry summer heat prevails in the Rhineland, and to avoid overheating in the newly built German Industry House in Cologne the windows have been double glazed with solar insulating glass type Gold 40/26 by Flachglas AG Delog-Detag, giving 40 per cent transmission of visible light rays with only 26 per cent of the solar energy entering the building

centration and the amount of dust in the atmosphere. Basically, half of all solar radiation is infra-red, lying outside the visible range.

\section{Requirements for Sun Insulating Glass}

For effective lighting through a sun-insulating glass, a high degree of opacity will be required in this area of infra-red radiation. Attenuation of infrared radiation must take place mainly by reflection and not by absorption, as the absorption of solar radiation leads to heating of the glass, which at a high temperature will transfer a considerable amount of the absorbed solar radiation into the room by convection and long-wave secondary radiation.

Also, glass designed to give protection from sunlight must conform to various requirements of light transmission. For example, in large open-plan offices with floor-to-ceiling glazing, the use of normal double-glazing with 80 per cent light transmission has little point; the window area would be too bright by comparison with the artificially lit area at the back of the room. On the Continent, in such cases, glass windows with a 30 to 40 per cent transparency are used; in the USA the transparency used is much lower, from 10 to 20 per cent. On the other hand, for other applications such as hospitals and schools, at least 50 per cent transmission is necessary.
Figure 2 shows the design of a glass unit coated to provide insulation from solar radiation. It is composed of two glass sheets forming a hermetically sealed unit with the metal-to-glass edges sealed by an adhesive or soldering process. The heat-reflecting film is applied to the inner surface of the glass which is on the outer wall of the building, and is thus protected from mechanical damage.

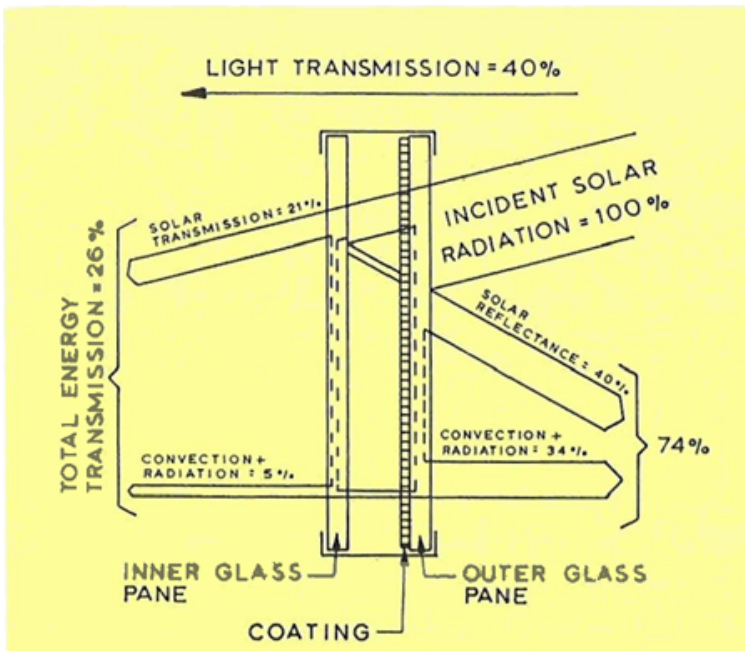

Fig. 2 Schematic representation of a gold-coated solar insulation double glazed unit with the corresponding distribution of solar energy 
The performance of this type of sun-insulating glass is best shown by an energy diagram such as Figure 2. This shows how much of the effective total solar radiation is directly reflected (solar reflection) and how much is transmitted (solar transmission). Then there is the secondary heat emission of the glass by convection and long-wave temperature radiation outwards and inwards, depending on the rise in temperature of the glass produced by the amount of solar radiation absorbed.

The total amount of heat reaching the interior of the room (total energy transmission) is therefore the sum of the solar transmission and the secondary emission into the room. Obviously the transparency of the glass (in relation to the sensitivity to light of the human eye) is also important. It is usual to give both figures in the form of a coefficient, transparency/ energy transmission. The figures shown in the energy diagram of Figure 2 relate to double-glazing with semi-transparent gold applied to a single glass pane.

\section{Simple Transparent Gold Films}

Effective sun-insulating glass for medium light transmission can be produced by a single gold deposit. Figure 3 shows the spectral transmission and reflectivity curves of an insulating glass unit treated with a gold film. Light transmission (as compared with the sensitivity to light of the human eye) is 40 per cent and total energy transmission 26 per cent.
This transmission is greatest in the blue-green area of the spectrum and decreases rapidly towards the infra-red area. To the same extent, reflectivity to long wave lengths increases up to 70 per cent. This means that the extinction of the infra-red radiation of the sun is due mainly to reflectivity and not to absorption.

Sun-insulating windows with such semi-transparent gold films have proved effective on account of their good optical properties. From inside, they appear light green in colour, but the tint is so slight as not to be unpleasant. Looked at from outside, buildings having these windows are impressive and colourful, as may be appreciated from the photograph on page 63.

Sun-insulating glass treated with a single gold film is, however, not effective if considerably more than 40 per cent transmission is required; the thickness of the gold film would have to be reduced. But as this thickness is reduced, so the film tends to lose its selective properties, that is its low infra-red transmission in relation to transparency (2). Even with the sun-insulating glass with very low light transmission (about 10 to 20 per cent) the effectiveness of these films is doubtful because gold films of this thickness would increase the intensity of the green colouring.

Then there are architectural considerations to be taken into account. From the architect's point of

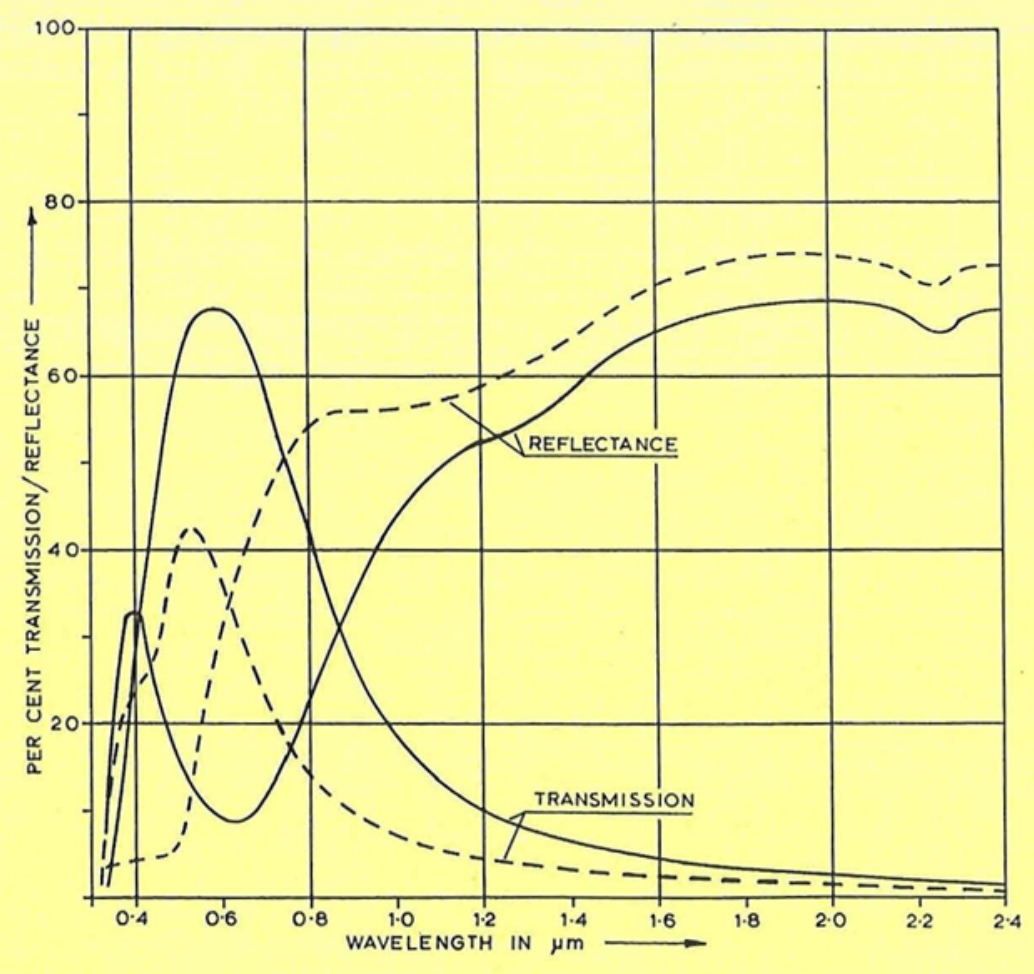

Fig. 3 Spectral transmission and reflectance of solar insulated double glazing

- - - Single gold film

Gold interference layer system

With the interference layer system the transmission of visible light rays is greatly increased by comparison with the single gold coating, while the reflectivity in the infra-red range is largely retained 
The new headquarters building of BMW in Muinich. This is glazed with a type of solar insulating glass, also by Flachglas AG Delog-Detag, that gives a blue reflection. The optical effect of the blue glass and the white building material-blue and white are the house colours of BMW - have a pleasing architectural effect. Solar insulating glass with various shades of blue, gold and bronzeo is available to provide scope for the architect in designing the façade

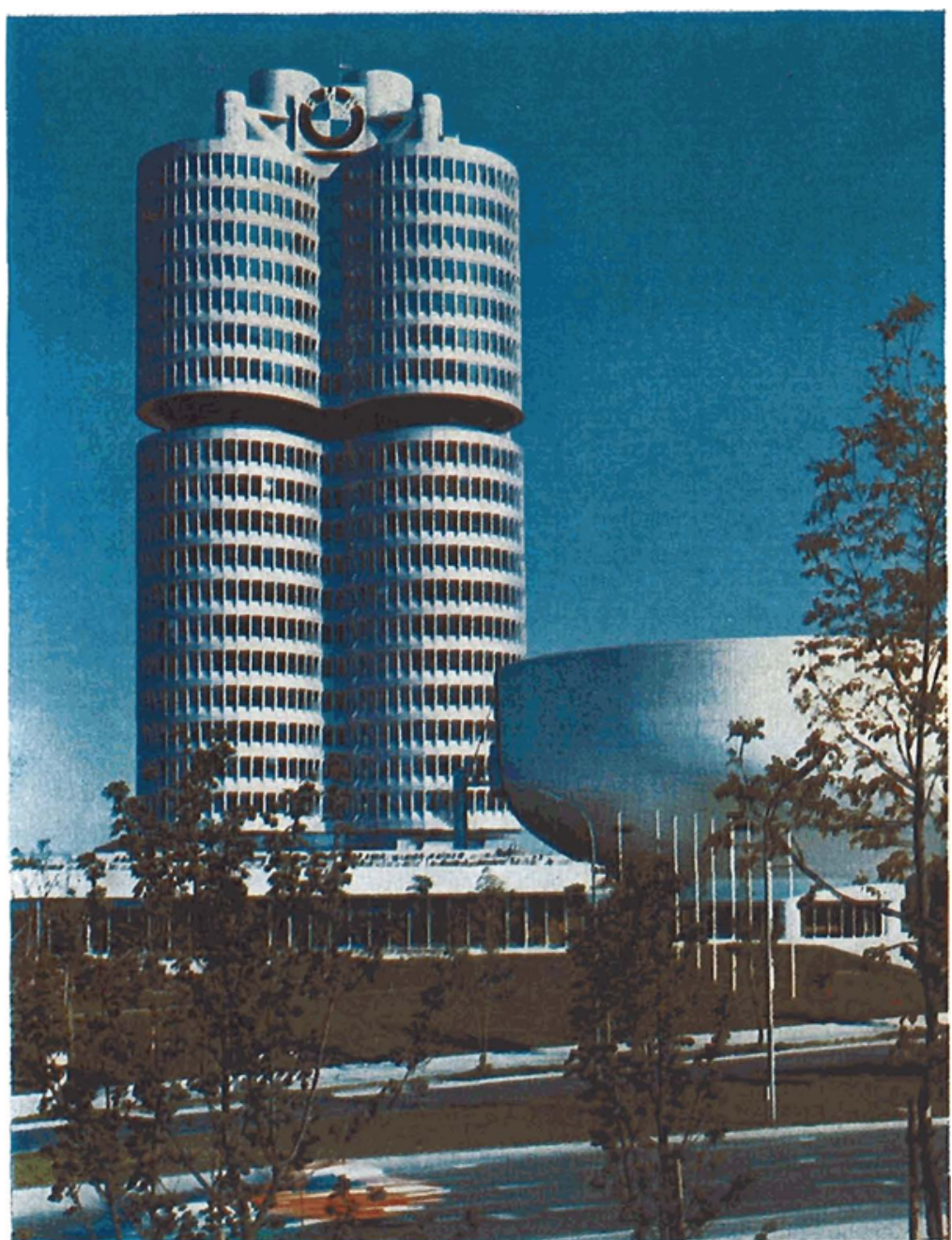

view, the exterior of the building should show a broad band of colour. This will combine with the structural lines of the building to give better proportions and give the architect greater scope in the design of the façade than can be achieved with a paler shade of gold.

\section{Gold Interference Layer Systems}

So the problem is one of changing the optical properties of the gold film in such a way as to retain high heat-reflectivity while greatly reducing visible reflectivity and increasing transmission, rendering it achromatic.

The method used is closely related to the wellknown process of reducing the reflectivity of glass surfaces used for instance in photographic apparatus and the blooming of instrument lenses. By applying thin dielectric films with a suitable refractive index and thickness, the reflected light rays at the surface and the interface of this additional film can be obtained with almost similar amplitudes but with a shift in the phase angle of $180^{\circ}$, so that they almost cancel each other out. In this case, since absorption is minimal, the non-reflected light intensity appears as enhanced transmission.

The high reflectivity of gold at long wavelengths is caused by the high electrical conductivity of the metal and this, in turn, by the number and mobility of its free s-electrons. A necessary condition is that the gold film is not made up of isolated "islands" but that it is homogeneous and continuous.

Methods of applying gold by firing on to the glass often do not produce continuous films, whereas vacuum-evaporated or vacuum-sputtered coatings on suitable substrates produce very low surface resistances of a few ohms per square with a 30 to 40 per cent transparency.

At shorter wave lengths, reflection and absorption behaviour become more complicated, at first in a narrow energy band by plasma-resonance caused by the interaction of free and bound electrons, followed by inter-band transference of $d$-electrons. In the case of gold, these phenomena lie in the middle of the visible spectrum; they are responsible for the 
yellow colour of gold. One can therefore say that the optical properties at long wave lengths are governed by the behaviour of the free electrons and at short wave lengths by the behaviour of the bound electrons. These complicated relationships are expressed mathematically by the optical constants $\mathrm{n}$ and $\mathrm{k}$ in the equation:

$$
\overline{\mathrm{n}}=\mathrm{n}-\mathrm{ik}
$$

where $\mathrm{n}$ stands for the dispersion factor and $\mathrm{k}$ for the absorption factor; for example, for a certain thickness of a gold film $n=1$ and $k=2$ if the wave length is $500 \mathrm{~nm}$. But it must be emphasised that these are not true constants but that both $\mathrm{n}$ and $\mathrm{k}$ are complex functions of wave length and film thickness. These functions have to be known in order to make quantitative calculations; at one time, writers differed considerably on the values of $n$ and $k$, but there has been a distinct improvement in recent years, due no doubt to improved methods of film application and more sophisticated methods of measurement.

But even if the optical constants are sufficiently exactly known, their mathematical processing is difficult and time-consuming. The solution to this problem consists in calculating the reflectivity values as functions of the wave length of layer systems, when, for instance, a transparent gold film of $200 \AA$ is deposited between two dielectric layers one of which is bonded to the glass substrate. Film thickness and refractive index of the dielectric layers have to be calculated so that the whole reflectivity in the visible spectrum is reduced to a minimum, while at the same time the reflection of heat radiation is brought to a high value. The necessary calculations are elementary, but as both complex phase angles and complex Frenel coefficients are involved, the resulting mathematical equations are cumbersome. Fortunately Kard (3) has proposed a method of calculation which provides a graphical means of evaluating the complex reflectivity factors.

Test results can be conveniently verified by evaporating a wedge-shaped film composed of the first dielectric material on to a glass substrate, applying to this layer a transparent film of gold of uniform thickness and evaporating on to this a second dielectric material, similarly wedge-shaped but so that the two wedges are at $90^{\circ}$ to each other. By this method one obtains a good overall impression of all possible thickness combinations of the two dielectric films and the required combination can be easily selected.

These layers do not show the normal colour of gold. For instance, in the blue-green spectral range they have a reflectivity value between 5 and 10 per cent, so that 70 to 75 per cent transmission can be expected. Compared with a single gold layer, the transmission maximum is shifted towards longer wave lengths on account of the interference layers. In transmitted light these films are slightly yellowish when looked through from the inside, giving clearer vision with greater contrast.

Gold is a metal that does not easily bond to oxide and silicate substrates. It is therefore expedient to
The Deutsche Lloyd office building in Stuttgart makes use of a type of gold-coated insulating glass that gives a highly reffective blue appearance from the outside. The cost of cooling this airconditioned building in summer has been greatly reduced by the installation of this form of solar insulation by Flachglas

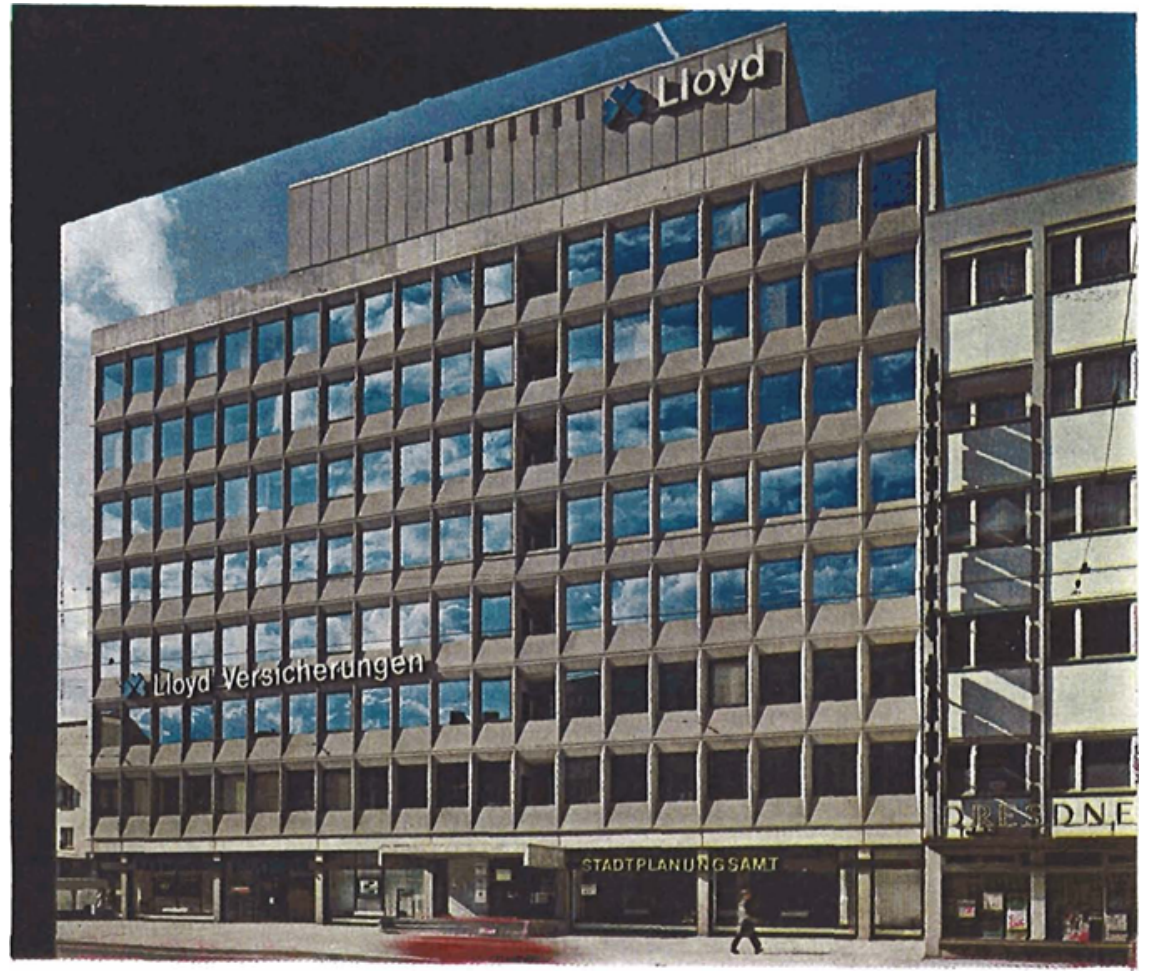




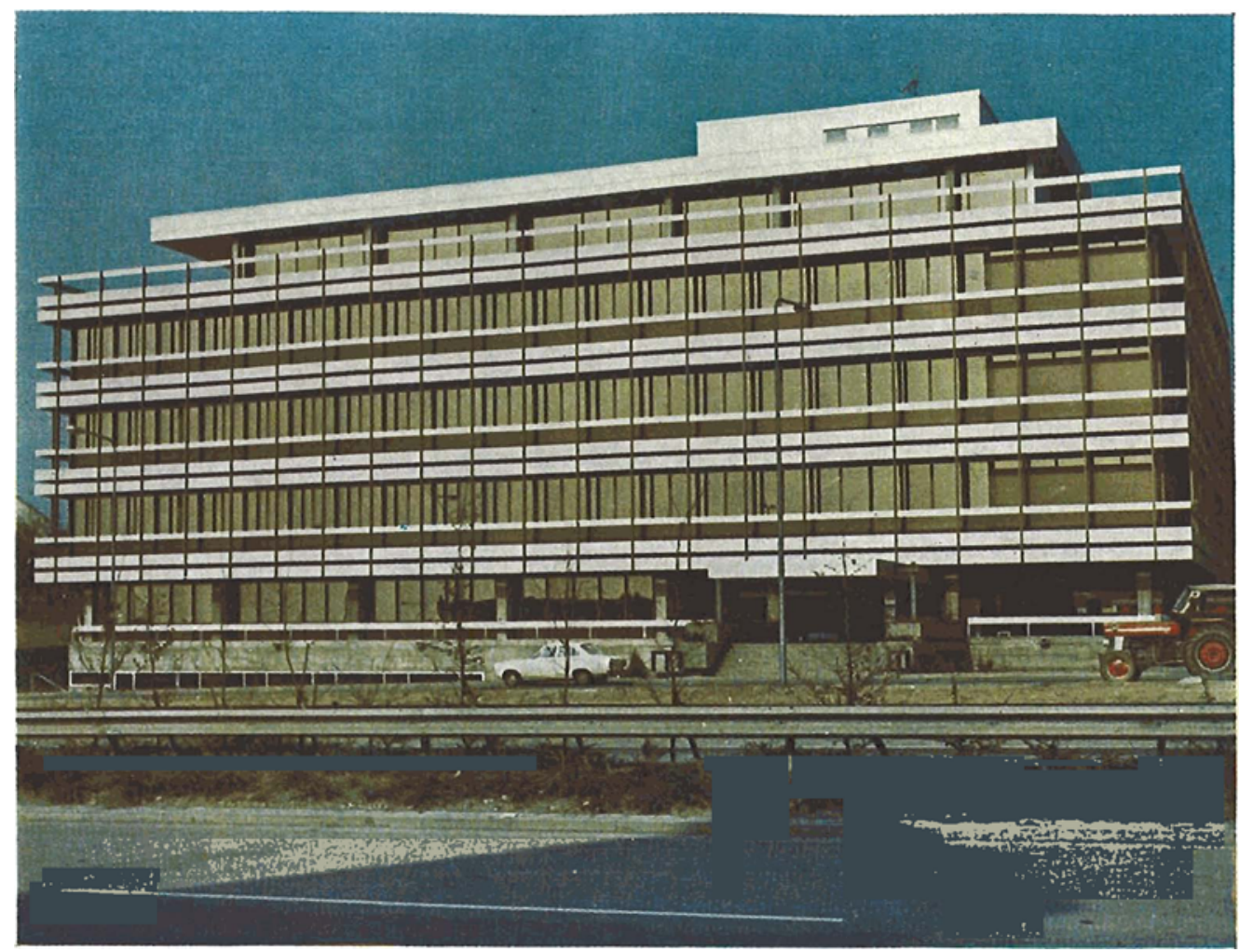

This imposing building is the headquarters of a firm of consultants in Wiesbaden. In this case the goldcoated insulating glass, also by Flachglas, has a soft bronze tone which contrasts effectively with the white facings of the constructional material

embed the gold film in dielectric layers to secure a satisfactory bond of the layers in the pack and good adherence of the pack to the glass substrate. This problem has now been solved and a process evolved which satisfactorily produces coated material for use as insulating glass. It is also possible, by using small additives to gold and by means of modern techniques, for instance electron beam evaporation, to apply 2 to $3 \mu \mathrm{m}$ glass layers on to the gold surface that are abrasion-resistant, so that they can be used satisfactorily when exposed to the atmosphere.

\section{Applications of Gold Interference Layer Systems}

By combining gold films with interference layers for sun-insulating glass, new applications have been developed, particularly in cases where high light transmission is needed.

Figure 3 shows the spectral transmission and reflectivity curves for a technical sun-insulating glass with a gold interference layer system of this type, and for comparison the corresponding curves for a product with a single gold film. Compared with the latter, the interference layers increase transmission in the visible range considerably. Transmission is increased from 40 to 66 per cent which is only 14 per cent less than that of a clear glass double-glazing unit ( 80 per cent). In the infra-red range, on the other hand, the reflectivity of the gold film is largely retained. That the reflectivity values differ somewhat for wavelengths of about $2 \mu \mathrm{m}$ for which the effect of the interference layering is almost nil is due to the fact that the thickness of the gold films is somewhat different in these products.

By its very nature, the total energy transmission, 44 per cent of the type with the interference layer, is higher than in the type with the single gold film. This is to a large extent governed by the greater heat caused by the higher light transmission engendered by solar radiation in the visible range. Looked at from outside, the typical gold effect is lost because of the anti-reflection effect of the interference layers; instead, they have a slightly blue appearance. The illustration on page 65 shows a building glazed with this type of glass.

In recent years developments in sun-insulating glass have tended to extend the colour palette so as to give architects more scope in designing the façades of buildings. Plate glass with varying shades of blue, gold or bronze, when looked at from the outside, is available. The building illustrated opposite has a highly reflective blue exterior in contrast with that shown on page 63 , while the building shown above has bronze-coloured glass. Light transmission lies between 66 and 22 per cent with the corresponding energy transmission between 44 and 15 per cent (4). 


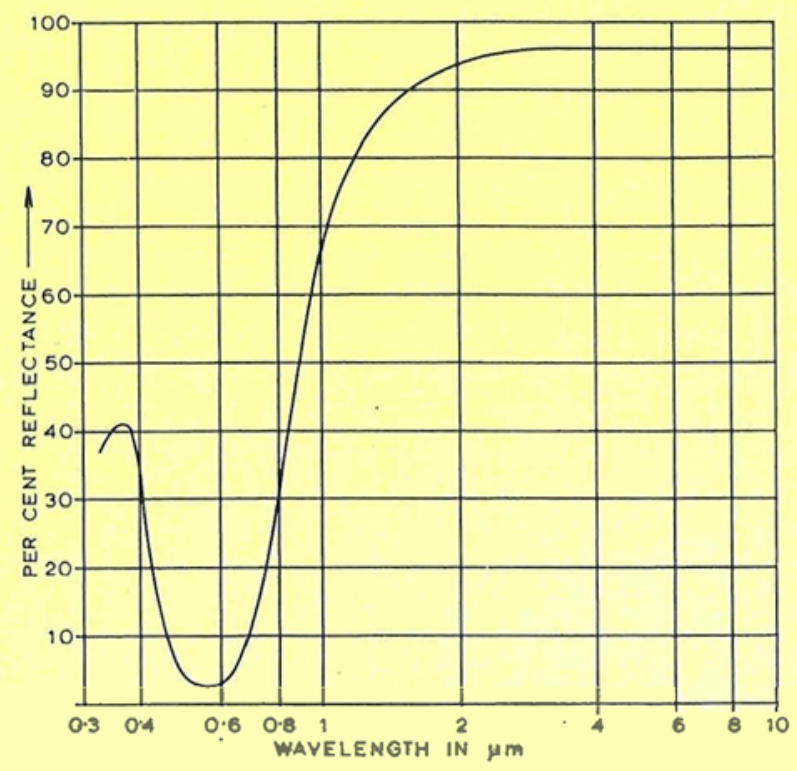

Fig. 4 An example of the spectral reflectivity of a gold interference coating system on the side of the glass exposed to the atmosphere

In all these cases the gold film is the actual selective filter element. Various technical values and different colours are obtained by a combination of the gold film with interference layers and alloy layers and the selection of the appropriate film thicknesses.

\section{Reduction of Heat Losses}

Double-glazing treated with a gold film has another advantage, apart from that of protection from solar radiation. Its heat transfer value is much lower than that of normal double-glazing. This means that in winter, with low outside temperatures, heating costs can be reduced by about 40 per cent.

A normal double glazing unit with the usual 12 $\mathrm{mm}$ air space has a heat loss of $2.6 \mathrm{kcal} / \mathrm{m}^{2} \mathrm{~h}^{\circ} \mathrm{C}$ compared with $5 \mathrm{kcal} / \mathrm{m}^{2} \mathrm{~h}^{\circ} \mathrm{C}$ for single glazing. This improvement is due to the additional heat insulation of the enclosed air space.

Heat transfer between the colder outer pane and the inner pane is due first to the thermal conductivity of the air space with additional convection and secondly to the radiation exchange between the two panes of glass. The radiation exchange with an air gap of $12 \mathrm{~mm}$ is about double that of the figure of thermal conductivity and convection.

This heat exchange is considerably lessened by the infra-red reflecting layer because the layered glass has a very low emissivity. The heat radiation of the glass panes corresponds quite well to the spectral energy distribution of a black body at approximately $300^{\circ} \mathrm{K}$ (room temperature) and includes the infra-red from about 4 to $30 \mu \mathrm{m}$ with a maximum at about $10 \mu \mathrm{m}$.

In this range of wave lengths, transparent gold films possess very high reflectivity. Figure 4 shows as an example the spectral reflectivity of a gold interference coating system on the side of the glass exposed to the atmosphere. In the visible range, reflectivity-including the effect of the interference layer-is very low and transmission correspondingly high (light transmission of the corresponding insulating glass pane $=66$ per cent). In the near infra-red range, reflectivity increases steeply, reaching more than 96 per cent in the $4 \mu \mathrm{m}$ wave length region. This corresponds to an emission figure of $\varepsilon=0.04$ compared with $\varepsilon=0.84$ (5) for non-layered window glass.

This coating reduces the heat exchange between the two window panes to almost nil, with a considerable increase in the heat resistance of the air space and a corresponding improvement in the heat loss. In all, heat loss, $2.6 \mathrm{kcal} / \mathrm{m}^{2} \mathrm{~h}^{\circ} \mathrm{C}$ for normal double glazing with an air space of $12 \mathrm{~mm}$ is reduced to $1.5 \mathrm{kcal} / \mathrm{m}^{2} \mathrm{~h}^{\circ} \mathrm{C}$. The degree of heat insulation resulting from the film is approximately equivalent to that given by a $30 \mathrm{~cm}$ thick wall of polished brick or tiles.

\section{Methods of Applying the Coating}

Vacuum-film methods are used exclusively, as they are the only ones which give continuous gold films without forming an "island" effect, a continuous film being essential for high infra-red reflectivity. The coating is carried out by cathode sputtering or by evaporation deposition methods. Processing large glass sheets up to an area of $10 \mathrm{~m}^{2}$ has been made possible by the development of large vacuum plants and by advances in coating techniques, thus ensuring a uniform thickness over such large areas.

Thus, in addition to its now well-established use for coating glass for heat protection in plants operating at very high temperatures, gold has found a new application in the building industry. Sun-insulating glass is also being increasingly used for the windows of air-conditioned trains on the Trans-Europa Expresses and on the Inter-City lines.

\section{References}

1 P. Moon, f. Franklin Inst., 1940, 230, 583

2 H. Schroder, Glastechn. Ber., 1966, 39, 156

3 P. G. Kard, Dokl. Akad. Nauk S.S.S.R., 1956, 108, 60

4 "Infrastop-Sonnenschutzgläser", Flachglas AG DelogDetag

5 J. I. Yellot, ASHRAE Fournal, 1964, (Jan.), 87 